\title{
Monoclonal Antibody and Peptide-Targeted Radiotherapy of Cancer
}

\author{
R.M. Reilly, ed. \\ Hoboken, NJ: John Wiley and Sons, 2010, 624 pages, \$149.95
}

Radioimmunotherapy and peptide-directed radiotherapy of solid tumors have been restricted by low tumor uptake; dose-limiting toxicity to normal tissues, including the bone marrow; and an intrinsic radioresistance. However, the success of radioimmunotherapy of non-Hodgkin lymphoma has proven that this approach is scientifically sound, translatable to clinical practice, and feasible. Continuing to gain momentum as a novel treatment strategy, targeted radiotherapy relies on and exploits the characteristics of malignant cells to attack tumors with radiation.

Thirty-five internationally well-recognized scientists have written this book to cover a range of diverse topics associated with targeted radiotherapy, including the optimization of biomolecule design and radiochemistry, cell and animal models for preclinical trials, radiation biology and dosimetry, and considerations in regulatory approval. This book consists of 17 chapters dealing with antibody engineering, radiochemistry of antibodies and peptides, radioimmunotherapy of radiolabeled peptides, peptide receptor radionuclide therapy, radioimmunotherapy of central nervous system tumors, lymphoma and leukemia, pretargeted radioimmunotherapy, Auger electron therapy, viral introduction of receptors, cell and tumor models, radiation biology, dosimetry, the bystander effect, evaluation of tumor response, economic attractiveness, and regulatory elements. Unique aspects of this book include a translational focus on development and evaluation of targeted radiopharmaceuticals for treating tumors, discussions on health economics issues and the utility of targeted radiotherapy, and new areas such as molecular imaging, the bystander effect, and combined radioimmunotherapy. This book delivers a wealth of information to push future discovery for targeted radiotherapy. A review of the current state of the art in the radioimmunotherapy field provides insight from today's cancer researchers as they reach for tomorrow's cures.

Most figures are black and white, but that appears to pose no problem in illustrating the key points. The tables and index are helpful, and the references are updated. This book is suitable for students and trainees in the field of radioimmunotherapy and for physicians in nuclear medicine, radiation, and medical oncology. The book will also be of interest to any research workers who look for new avenues to more effective killing of cancer cells.

\section{E. Edmund Kim}

M.D. Anderson Cancer Center

1515 Holcombe Blvd., Unit 1264

Houston, TX 77030

E-mail: ekim@di.mdacc.tmc.edu 